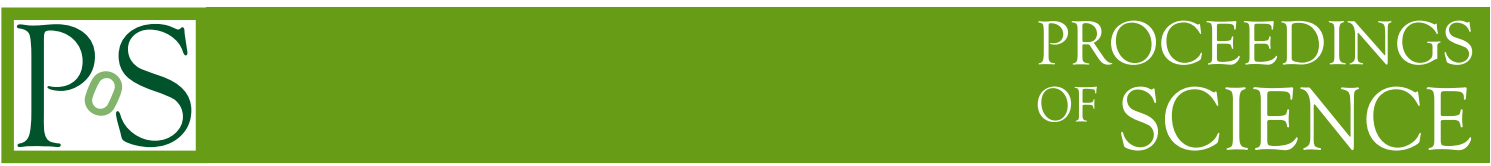

\title{
High-energy cosmic ray modulation associated with interplanetary shocks observed by the GMDN
}

\author{
Carlos Roberto Braga* $a$, Rafael R. S. de Mendonça $a$, Ezequiel Echer $a$, Alisson Dal \\ Lago $a$, Ana Clara S. Pinto $a$, Kazuoki Munakata $b$, Takao Kuwabara $c$, Masayoshi \\ Kozai $d$, Chihiro Kato $b$, Nelson J. Schuch $e$, Marlos Rockenbach $a$, Hala K. Al \\ Jassar $f$, Madan M. Sharma $f$, Munetoshi Tokumaru $g$, Marc L. Duldig $h$, John E. \\ Humble $h$, Paul A. Evenson $i$, and Ismail Sabbah $j$ \\ a Space Geophysics Division, National Institute for Space Research \\ São José dos Campos, SP, 12227-010, Brazil \\ b Physics Department, Shinshu University \\ Matsumoto, Nagano, 390-8621, Japan \\ c Graduate School of Science, Chiba University \\ Chiba city, Chiba, 263-8522, Japan \\ $d$ Institute of Space and Astronautical Science, Japan Aerospace Exploration Agency \\ Sagamihara, Kanagawa, 252-5210, Japan \\ e Southern Regional Space Research Center, National Institute for Space Research \\ Santa Maria, RS, 97105-900, Brazil \\ $f$ Physics Department, Kuwait University \\ Kuwait City, 13060, Kuwait \\ $g$ Institute for Space-Earth Environmental Research, Nagoya University \\ Nagoya, Aichi, 464-8601, Japan \\ $h$ School of Physical Sciences, University of Tasmania \\ Hobart, Tasmania, 7001, Australia \\ i Bartol Research Institute, Department of Physics and Astronomy, University of Delaware \\ Newark, DE 19716, USA \\ j Department of Natural Sciences, College of Health Sciences, Public Authority of Applied \\ Education and Training \\ Kuwait City, 72853, Kuwait \\ E-mail: carlos.braga@inpe.br
}


Interplanetary shocks are caused both by interplanetary counterparts of coronal mass ejections (ICMEs) and by co-rotating interaction regions (CIRs) propagating in the interplanetary medium. CIRs are formed by the interaction between high-speed and slow solar wind streams. When the interplanetary disturbance propagates faster than the magnetosonic wave speed, in the solar wind frame, a shock wave is formed. Shocks frequently produce decreases of cosmic rays observed both by neutron monitors and muon detectors located at the Earth's surface. In this work, we analyze this kind of modulation of high-energy cosmic rays $(\gtrsim 50 \mathrm{GeV})$ observed by the Global Muon Detector Network (GMDN). After correcting both the atmospheric temperature and pressure effects, we calculated the isotropic intensity and the anisotropy vector. From a list of 38 interplanetary shocks identified in 2015 using interplanetary magnetic field and plasma parameters, we performed a superposed epoch analysis grouping the events by type and orientation of shocks. We found that the cosmic ray isotropic intensity is higher when it is associated to fast forward shocks when compared to fast reverse shocks. We also identified some differences in the anisotropy vector when comparing different types of shocks or shocks that are quasi-perpendicular with the remaining ones. 


\section{Introduction}

When an interplanetary disturbance propagates faster than the magnetosonic wave speed, in the solar wind frame, a shock wave is formed [1]. Due to the turbulent field region generated behind a shock, high-energy cosmic rays are modulated and a decrease is frequently registered by neutron monitors and muon detectors [2].

The type of shock most commonly observed in the interplanetary medium are the fast forward (FF) shocks. When those shocks are observed near the earth, they are generally associated to interplanetary counterparts of coronal mass ejections (ICMEs) [3]. The time profile of FF shocks show increase in the interplanetary magnetic field, the solar wind speed, temperature and density.

Other category of shocks propagates toward the Sun (instead of away from it, as the FF and the solar wind do). These shocks are called fast reverse (FR) shocks. They are mainly observed at the boundaries of co-rotating interaction regions (CIR), regions formed by the interaction of fast and slow solar wind streams $[4,5]$. Some authors identify structures similar to CIR but with a more relaxed criteria regarding the duration of the event and call them Streaming Interaction Regions (SIR) (see, e.g., [6]). While the CIRs persist for successive Carrington Rotations (CR), the SIRs do not have this criterion. CIRs frequently are associated with a pair of shocks at $1 \mathrm{AU}$ : a FF at the beginning and a FR at its end [7]. FR are sometimes observed at the end part of fast expanding ICMEs $[8,9]$. When inspecting the time-profile of the interplanetary parameters, the FR shocks show increase in the solar wind speed and decrease in the interplanetary magnetic field, solar wind density and temperature.

Other category of shocks are the slow shocks that can be either forward or reverse. Shocks of these categories, however, are rarely observed in the interplanetary medium close to the Earth [10] and will not be discussed in this article.

In this work we study the relation between the shock type and the isotropic cosmic ray decrease observed in the $\mathrm{GeV}$ range by muon detectors during a set of interplanetary shock waves of different types in order to compare possible differences between them. We also study the relation between the shocks type and the anisotropy vector.

\section{Observation and data analysis}

We use the high-energy ( $\gtrsim 50 \mathrm{GeV}$ ) cosmic ray data observed by the Global Muon detector Network (GMDN), a network formed by 4 muon detectors installed in Nagoya (Japan), Hobart (Australia), São Marinho da Serra (Brazil) and Kuwait City (Kuwait) [11].

The relation between CIRs observed from 2001 to 2004 and cosmic ray decrease observed by one of the muon detectors from the GMDN was performed in a previous study but the shock observation and type during these events was not taken into account [12].

A previous study related the cosmic ray decreases associated with shocks were already performed using observations from the GMDN up to 2014 (see, e.g., [13]) and the events were selected using the geomagnetic storm sudden commencement (SSC) listed by the German Research Centre for Geosciences (GFZ) as the identification of the shock arrival at Earth. Although the SSCs are considered to be caused by the interplanetary shocks [14], cosmic rays are not directly modulated by the geomagnetic field conditions but primary by the conditions in the interplanetary magnetic 
field. For this reason, we decided to use a shock list identified using observations from the interplanetary medium rather than the SSC events.

Before starting the analysis of the effects of the interplanetary phenomena in the cosmic ray data, we removed the contributions from the atmospheric effects. The correction of the pressure effect follows the method described in [15].

As for the temperature effect, we adopted the mass weighted method (see, e.g., [16] and [17]. We have chosen this method because it was found to be the best to remove the temperature effects on the GMDN data among several other methods available [18]. We used the temperature profiles extracted from the Global Data Assimilation System (GDAS). The data consist of the temperature at isobar levels ranging from 20 to $1000 \mathrm{hPa}$ provided every 3 hours. The data is available online at ftp://arlftp.arlhq.noaa.gov/archives/gdasl/

In order to understand the cosmic ray modulation caused by shocks, we calculated the cosmic ray isotropic intensity and the anisotropy vector. Briefly, the isotropic intensity is the decrease in the primary cosmic ray free from local geomagnetic effects. The anisotropy points toward the direction that has the maximum cosmic ray streaming. A detailed of the methodology can be found in [19].

We took the shocks identified by the Heliospheric Shock Database (http://ipshocks . fi) observed by the WIND spacecraft for the period of 2015 only. Beyond the observation time, we took two other parameters derived for each event: the shock type and the shock normal (the angle between the shock normal and the interplanetary magnetic field). The shock normal was derived using observations from both the WIND spacecraft in the Geocentric Solar Ecliptic (GSE) coordinate system. The method used to derive the shock is the mixed-mode method from [20].

\section{Results}

The Heliospheric Shock Database report a total of 38 shocks observed in 2015 according to the observations from WIND spacecraft. Among them, 28 are FF and 10 FR. The ratio of FF and FR shock is similar to the one found in previous works at solar minimum [10]. The comparison of the isotropic intensity observed during both types of shocks in shown in Figure 1 using a superposed epoch analysis. We have used the shock observation hour as the time reference of each shock. The mean hourly isotropic intensity in indicated in black for the FF shocks and in blue for the FR shocks. The error bars indicate the standard error of the mean. It is clear that after the shock observation, the cosmic ray intensity decreases with higher intensity when considering the FF shocks. Other difference is the duration of the decrease: while the mean of the intensity goes to zero approximately 48 hours after the FR observation, the decrease is kept for the FF at least up to 48 hours.

We also compared the anisotropy vector (after correction of the solar wind effect) in the twoday periods before and after the shock event (Figure 2). To each anisotropy component, we subtracted the mean anisotropy of the same component at the moment of the shock observation so that the mean of all components are set to zero at the shock observation time. We can notice a clear difference in the component of the anisotropy in the $x$ direction. While it is approximately constant for the FF shocks, it decreases on the 48-hour period before the FR shocks. 


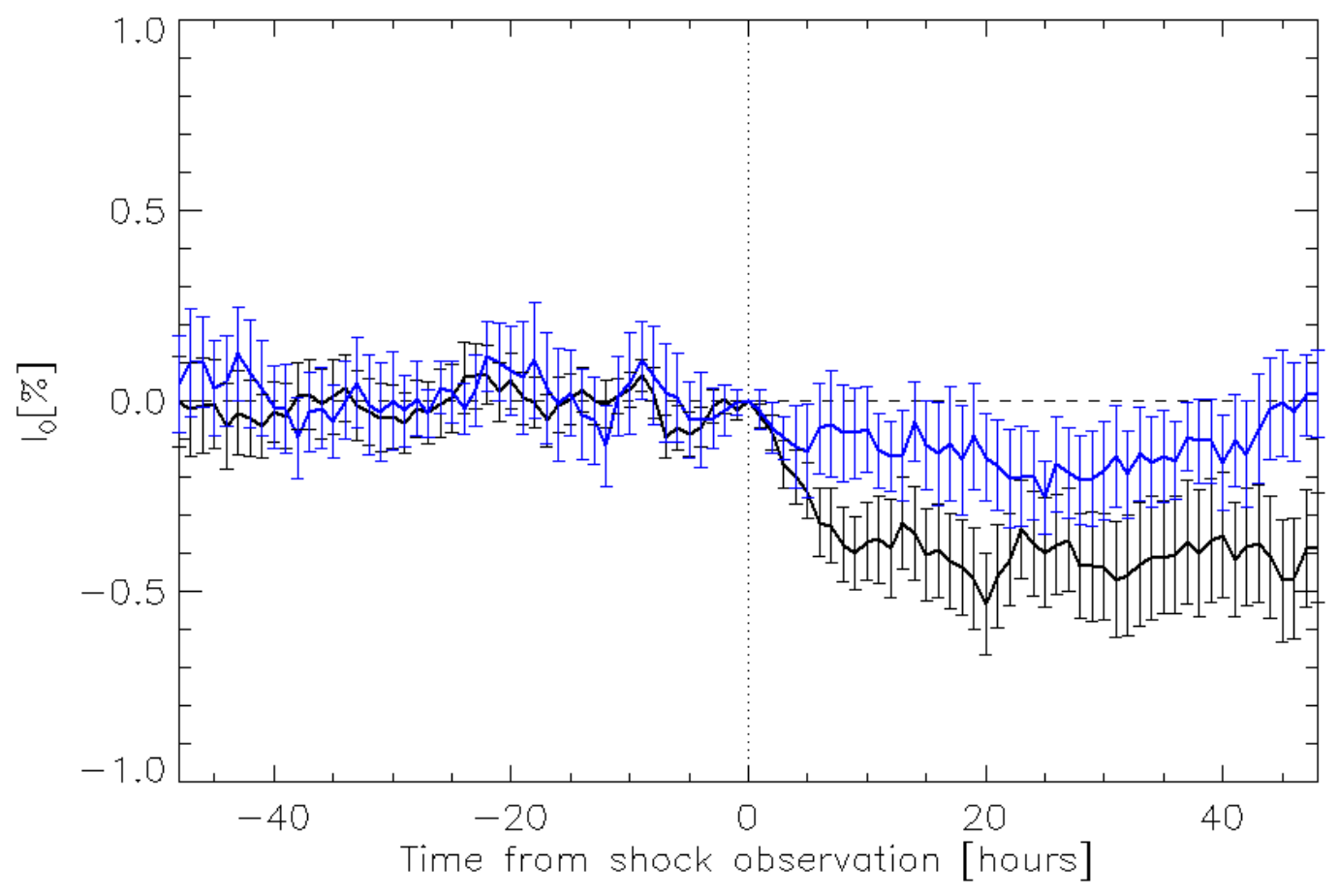

Figure 1: Comparison of the cosmic ray intensity decrease observed at Fast Forward (represented in black) and Fast Reverse (represented in blue) interplanetary shock Waves.

We compared the anisotropy for groups of shocks with different ranges of the angle theta between the shock normal and the interplanetary magnetic field direction upstream of the shock. Since the number of FR shocks are quite small, we took only the 28 FF in this analysis. We separated them into two categories: $\theta<60^{\circ}$ and $\theta \geqslant 60^{\circ}$. We have chosen the limit of $60^{\circ}$ because it forms two groups so that each one has more than 10 events, avoiding having a category with very reduced number of events that could lead to high errors in the calculation of the mean of the category. The result of this comparison is shown in Figure 3. As they are, the error in the mean anisotropy values of each component is approximately $0.1 \%$.

We found that specially in the 48-hour period before the shock observation, FF shocks with $\theta<60^{\circ}$ have significant smaller values of the $y$ component of the anisotropy when compared to the remaining FF shocks $\left(\theta \geqslant 60^{\circ}\right)$. In other words, the shocks that are quasi perpendicular do not have a significant $y$ anisotropy. There are also some differences on the remaining anisotropy components with smaller amplitude.

One point that must be taken into consideration with this analysis is that the interplanetary shocks are frequently observed ahead or after interplanetary structures such as ICMEs and CIRs. In this way, the modulation of the cosmic ray shown here is not limited to the shock itself but possibly includes effects from the associated interplanetary phenomena. For instance, ICMEs frequently follow FF shocks. Therefore, the differences found when comparing FF and FR shocks and FF shocks with different $\theta$ could be related at least partially to the cosmic ray modulation associated to the interplanetary structures that are associated to each group of shock events discussed here. 

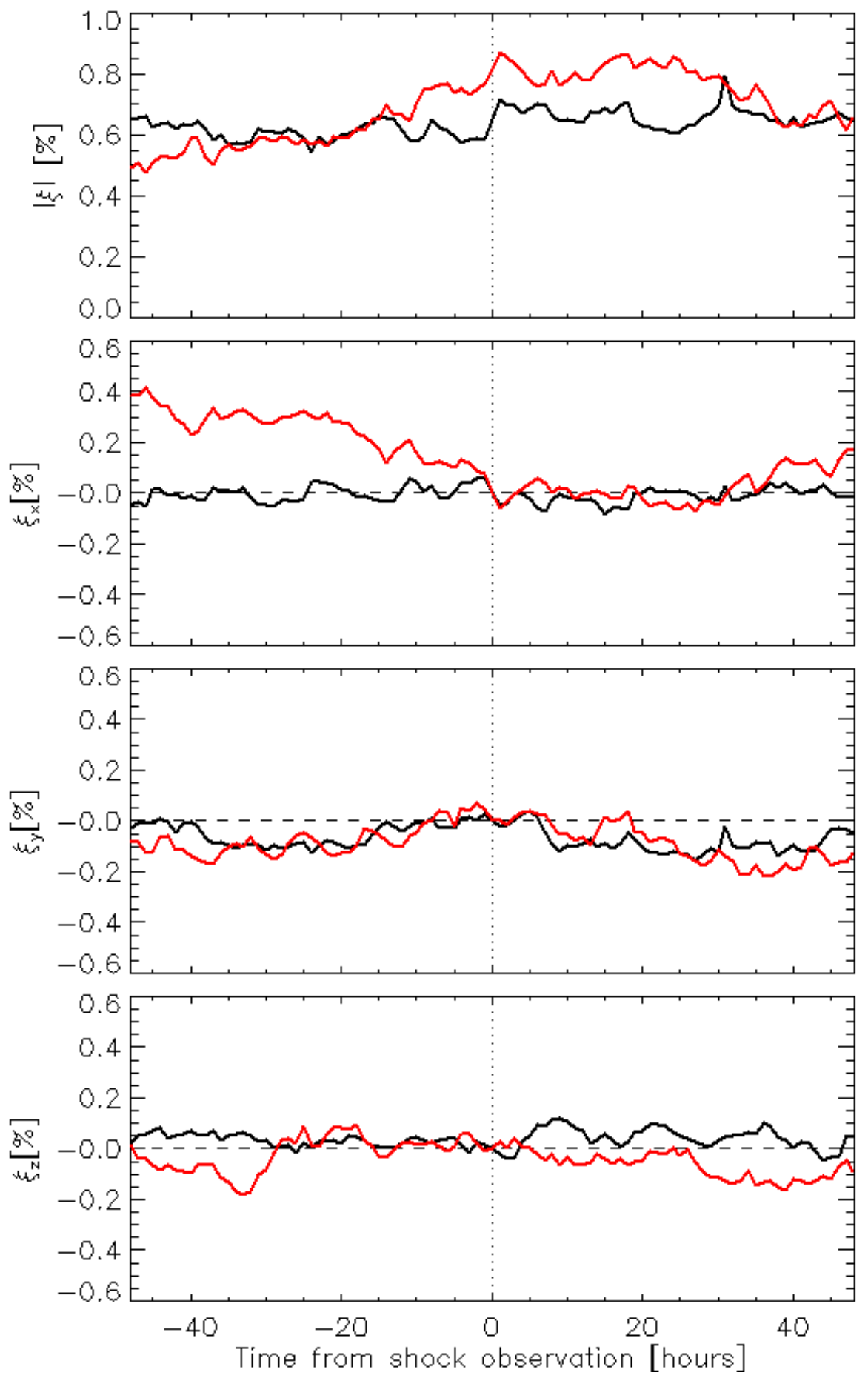

Figure 2: Comparison of the cosmic ray anisotropy observed at Fast Forward (represented in black) and Fast Reverse (represented in red) interplanetary shock Waves. 

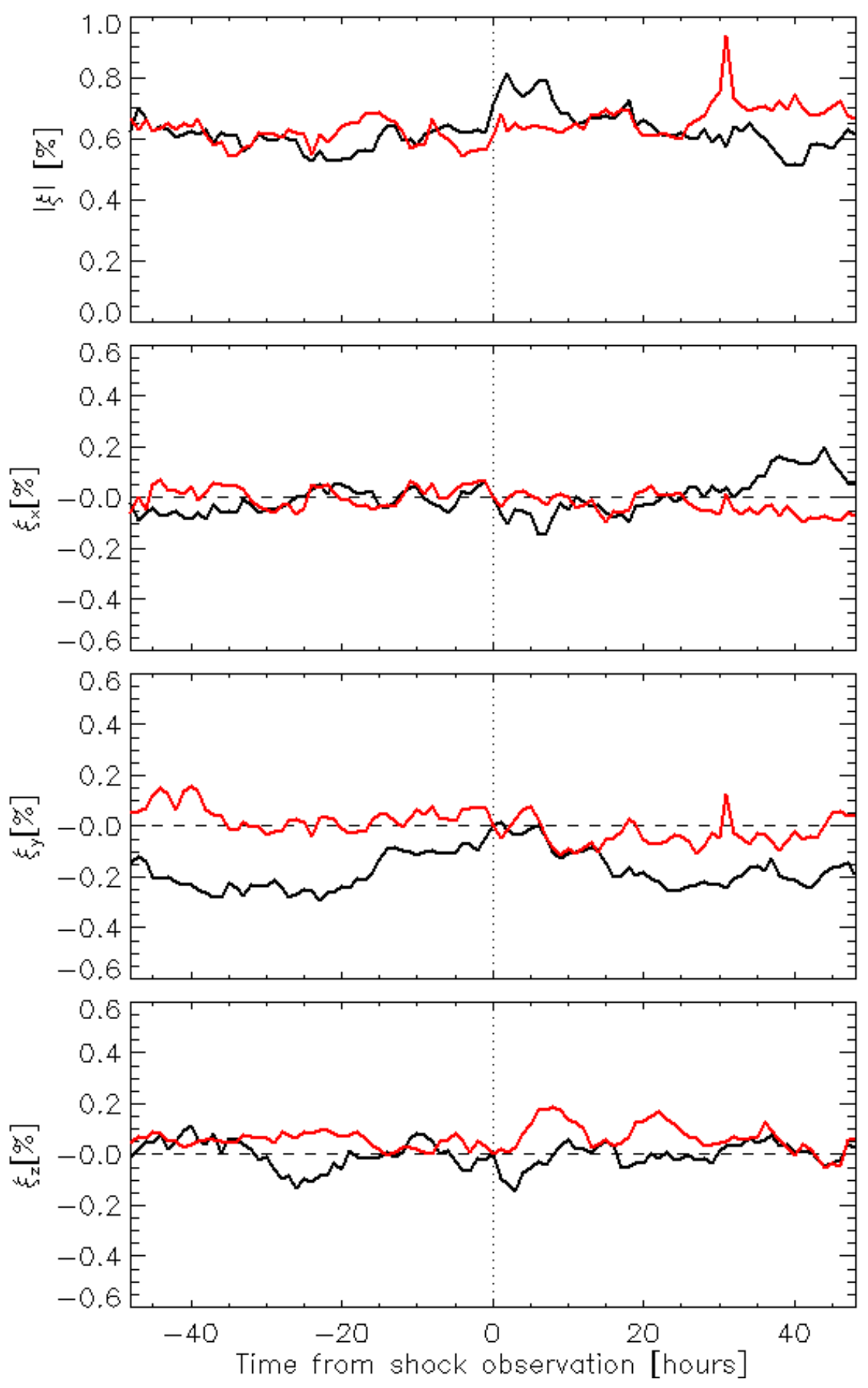

Figure 3: Comparison of the cosmic ray anisotropy observed at Fast Forward in two different ranges of angle between the shock normal and the magnetic field: the quasi perpendicular $\left(\theta \geqslant 60^{\circ}\right)$ are represented in red and the remaining in black. 


\section{Final remarks}

We have studied the cosmic ray anisotropy and isotropic intensity observed by the Global Muon Detector Network (GMDN) during periods of observation of interplanetary shocks. When comparing the average of the events observed in 2015, we found that the cosmic ray isotropic intensity decrease is higher when it is associated to fast forward shocks when compared to fast reserve shocks. In this period, there are no slow shocks reported. We also found that the anisotropy components of the cosmic ray decrease are significantly different when comparing quasi-perpendicular FF shocks with the remaining FF shocks.

In future work, we intend to extend the analysis for the full period of the data observed by the GMDN up to now (available since 2006) in order to compare whether the current observations were a general behavior through a full solar cycle or if they are specific for the events observed in the period analyzed here. Several other parameters related to the shock are intended to be compared with the cosmic ray anisotropy such as the ratio between magnetic field, solar wind proton density upstream and downstream of the shock wave.

\section{Acknowledgments}

C. R. Braga acknowledges grants \#2014/24711-6, \#2013/02712-8 and \#2012/05436-9 from São Paulo Research Foundation (FAPESP). A. Dal Lago acknowledges CNPq for grant 304209/20147. E. Echer acknowledges CNPq for grant 302583/2015-7. R. R. S. de Mendonça acknowledges and CNPq for grant 152050/2016-7. This paper uses data from the Heliospheric Shock Database, generated and maintained at the University of Helsinki.

\section{References}

[1] E. N. Parker. "Sudden Expansion of the Corona Following a Large Solar Flare and the Attendant Magnetic Field and Cosmic-Ray Effects.” In: 133 (May 1961), p. 1014. DOI: $10.1086 / 147105$.

[2] Hilary V. Cane. "Coronal Mass Ejections and Forbush Decreases". In: Space Science Reviews 93.1 (2000), pp. 55-77. ISSN: 1572-9672. DOI: 10 . 1023 / A : 1026532125747. URL: http://dx.doi.org/10.1023/A:1026532125747.

[3] N. R. Sheeley et al. "Coronal mass ejections and interplanetary shocks". In: Journal of Geophysical Research: Space Physics 90.A1 (1985), pp. 163-175. ISSN: 2156-2202. DOI: 10 . 1029/JA090iA01p00163. URL: http://dx.doi.org/10.1029/JA090iA01p00163.

[4] Leonard F. Burlaga. Interplanetary magnetohydrodynamics. Oxford University Press on Demand, 1995.

[5] A. S. Krieger, A. F. Timothy, and E. C. Roelof. "A coronal hole and its identification as the source of a high velocity solar wind stream”. In: Solar Physics 29.2 (Apr. 1973), pp. 505525. ISSN: 1573-093X. DOI: 10.1007 /BF0 0150828 . URL: https://doi.org/10 . $1007 / \mathrm{BF} 00150828$. 
[6] L. K. Jian et al. "Multi-Spacecraft Observations: Stream Interactions andfffdfffdAssociated Structures". In: Solar Physics 259.1 (2009), p. 345. ISSN: 1573-093X. DOI: 10 . 1007 / s11207-009-9445-3. URL: http://dx.doi.org/10.1007/s11207-0099445-3.

[7] E. Echer, B.T. Tsurutani, and F.L. Guarnieri. "Forward and reverse CIR shocks at 4 - 5AU: Ulysses”. In: Advances in Space Research 45.6 (2010), pp. 798-803. ISSN: 0273-1177. DOI: http://dx.doi.org/10.1016/j.asr.2009.11.011. URL: http://www. sciencedirect.com/science/article/pii/s0273117709007145.

[8] J. T. Gosling et al. "A forward-reverse shock pair in the solar wind driven by over-expansion of a coronal mass ejection: Ulysses observations". In: Geophysical Research Letters 21.3 (1994), pp. 237-240. ISSN: 1944-8007. DOI: 10 . 1029 / 94GL00001. URL: http: / / dx.doi.org/10.1029/94GL00001.

[9] Y. C. Whang. "Forward-reverse shock pairs associated with coronal mass ejections". In: Journal of Geophysical Research: Space Physics 93.A6 (1988), pp. 5897-5902. ISSN: 21562202. DOI: 10.1029 /JA093iA06p05897. URL: http://dx.doi.org/10.1029/ JA093iA06P05897.

[10] Ezequiel Echer et al. "Interplanetary shock parameters during solar activity maximum (2000) and minimum (1995-1996)”. In: Brazilian Journal of Physics 33.1 (2003), pp. 115-122.

[11] M. Rockenbach et al. "Global Muon Detector Network Used for Space Weather Applications”. In: Space Science Reviews 182.1 (2014), pp. 1-18. ISSN: 1572-9672. DOI: 10 . 1007/s11214-014-0048-4. URL: http://dx.doi.org/10.1007/s11214014-0048-4.

[12] M.R. Da Silva et al. "Muon and neutron observations in connection with the corotating interaction regions". In: Advances in Space Research 40.3 (2007), pp. 348-352. ISSN: 0273-1177. DOI: http://dx.doi.org/10.1016/j.asr.2006.12.013. URL: http: / / www.sciencedirect.com/science/article/pii/s0273117706007721.

[13] M. Kozai et al. "Average Spatial Distribution of Cosmic Rays behind the Interplanetary Shock - Global Muon Detector Network Observations". In: The Astrophysical Journal 825.2 (2016), p. 100. URL: http://stacks.iop.org/0004-637X/825/i=2/a=100.

[14] E. J. Smith. "Observations of interplanetary shocks: Recent progress". In: Space Science Reviews 34.1 (1983), pp. 101-110. ISSN: 1572-9672. DOI: 10 . 1007 /BF 00221200 . URL: http://dx.doi.org/10.1007/BF00221200.

[15] Lucas R. Vieira et al. "Near 13.5-day periodicity in Muon Detector data during late 2001 and early 2002". In: Advances in Space Research 49.11 (2012). Advances in theory and observation of solar system dynamics - I, pp. 1615-1622. ISSN: 0273-1177. DOI: http : / / dx . doi.org/10.1016/j.asr.2012.01 .017. URL: http : / / www . sciencedirect.com/science/article/pii/s0273117712000580. 
[16] V. L. Yanchukovsky, G. Ya. Filimonov, and R. Z. Hisamov. "Atmospheric variations in muon intensity for different zenith angles". In: Bulletin of the Russian Academy of Sciences: Physics 71.7 (2007), pp. 1038-1040. ISSN: 1934-9432. DOI: 10.3103 /S1 $06287380707043 X$. URL: http://dx.doi.org/10.3103/S106287380707043X.

[17] M. D. Berkova et al. "Temperature effect of the muon component and practical questions for considering it in real time". In: Bulletin of the Russian Academy of Sciences: Physics 75.6 (2011), pp. 820-824. ISSN: 1934-9432. DOI: 10 . 3103 / S1062873811060086. URL: http://dx.doi.org/10.3103/S1062873811060086.

[18] R. R. S. de Mendonça et al. "Temperature Effect in Secondary Cosmic Rays (MUONS) Observed at the Ground: Analysis of the Global MUON Detector Network Data". In: The Astrophysical Journal 830.2 (2016), p. 88. URL: http: / / stacks . iop. org / 0004 $637 X / 830 / i=2 / a=88$.

[19] Carlos Roberto Braga. "Study of coronal mass ejections and their corresponding interplanetary structures using combined observations of cosmic ray detectors and coronagraphs". en. PhD thesis. São José dos Campos: Instituto Nacional de Pesquisas Espaciais (INPE), Feb. 2015, p. 326. URL: http://urlib. net/sid. inpe.br/mtc-m21b/2015/01. 30.19 .09$.

[20] B. Abraham-Shrauner and S. H. Yun. "Interplanetary shocks seen by Ames Plasma Probe on Pioneer 6 and 7". In: Journal of Geophysical Research 81.13 (1976), pp. 2097-2102. ISSN: 2156-2202. DOI: 10 .1029/JA081i013p02097. URL: http://dx.doi .org/10 . 1029/JA081i013p02097. 